\title{
Enhancing Quality of Lossy Compressed Images using Minimum Decreasing Technique
}

\author{
Ahmed L. Alshami, Prof. Mohammed Otair \\ Amman Arab University \\ Faculty of Computer Science and Informatics \\ Amman, Jordan
}

\begin{abstract}
The acceleration in technology development came with the urgent need to use large amounts of information, and the way of storing or transferring the huge information via various digital networks became very important issues, particularly in terms of compressing size and quality preservation. The digital image usage has become widespread in many sectors; it become important to manipulate these images to use them in optimal form by maintaining the quality, especially during the compression process using the lossy techniques. This paper presents a new technique to enhance the quality of compressed images while preserving the compression ratio by adding some of pre-processing steps before implementing any existing lossy compression technique. The proposed technique depends on decreasing the minimum elements from the image pixels values in each: row, column, and $2 \times 2$ block, respectively. These steps minimize the required number of bits to represent each pixel in the compressed images. In order to prove the efficiency of the proposed technique, two lossy compression techniques (novel and classical) were implemented with the proposed. They implemented on wide range of well-known images with different dimensions, sizes, and types. The experimental results show that the quality of the decompressed images with the proposed technique were enhanced in terms of: MSE, MAE and PSNR as quality evaluation metrics.
\end{abstract}

Keywords-Image compression; lossy technique; lossless technique; image quality measurement; RIFD and JPEG

\section{INTRODUCTION}

The importance of the digital images and transmitting them along all networks either wired or wireless is increasing dayby-day. These images have very huge sizes in general and need to be compressed in order to accelerate transmitting process. As the digital image became widely used, image processing became a necessary field in many areas and for numerous reasons such as in medical imaging, social media, communications and security cameras. In the area of digital image processing the input for each process is an image, where the output can be an image or a certain attribute or information associated with the original or processed image that results after particular one or several processes applied on the original image [1].

Image compression is a field of image processes which means minimizing the size of the images file without effect on the quality of the image. Therefore, the aim of the compression is reducing the image size that allows the amount of disk or memory space to store more images. In addition, it reduces the time required for images to be sent or downloaded over the Internet [2].
The compression techniques are categorized into Lossless and Lossy. The first one has a good performance on the quality of the compressed images with no loss of any part of the images. However, when the researchers evaluate these techniques in terms of the compression ratio, they found that those techniques have very low performance comparing with the lossless techniques. On the other hand, lossy techniques have high distortion rates. So, most of the current researchers in this field try to find a good level of hashing both techniques, preserving or improving the compression ratio and reducing the distortion.

This paper tries to enhance the quality compressed images of the lossy compression techniques; it proposes a preprocessing lossless procedure prior to any lossy technique which produces a hybrid technique. In order to prove the achievability and efficiency of the proposed pre-processing technique, two lossy techniques were selected which are: the classic JPEG compression technique and the novel lossy technique RIFD [3]. The proposed technique can be applied generally on the digital images whether it is colored or gray scale with different bit-depth values (i.e. number of bits to represent the pixel values). It provides good compression ratio and lower distortion values, by taking the best features from the lossy and lossless techniques that has been used. Moreover, this paper aimed to reduce the bit-depth along with reducing the compression distortion and then improving the quality of the comparison process as a whole.

The rest of the paper is organized as follows: Section 2 introduces a general overview of the mostly related works. Section 3 describes the objective quality evaluation metrics that used in the experiments. The Proposed Technique which is called Minimizing Decreasing Technique (MDT) will be explained in Section 4. Section 5 discusses the experiments and the results of the paper. Finally, the paper is concluded in Section 6.

\section{RELATED WORK}

In the literature, the compression techniques can be mainly divided into two types: Lossy and Lossless, each one of them has its own positive characteristics. The priority of the lossy technique is the high compression ratio but with a percentage of distortion, while the lossless techniques compression produce a low rate of compression ratio without any distortion. This section presents some of the literature reviews that are related to the core subject of this study.

The researchers in [4] indicate that data can be compressed by decreasing the redundancy in the main data, but this makes 
the data have further errors. In this study a novel method of an image compression depend on a different technique which has been formed for image compression which has been called Five Modulus Method (FMM). The new procedure consists of converting every pixel value in an $8 \times 8$ block into a several of 5 for every of the R, G and B arrays. Then the new values can be divided by 5 to have new 6-bit length pixel values, and it has lower storage area than the original image. This study offered a new compression order of the new values as a stream of bits, which improved the chance for storing the new compressed image easily. This study explains the potential of the FMM depend on image compression technique, the priority of this method is the high PSNR even though it has low compression ratio. This technique is appropriate for bi-level images, where the pixel is symbolize by one byte (8-bit). Because of the low compression ratio, this method cannot be used as a standalone technique, but it could add as scheme within other compression techniques.

Zhou et al. [5] proved a new image compression encryption hybrid algorithm based on compressive sensing and random pixel exchanging, where the compression and the encryption are completed simultaneously, where the key is easily distributed, stored or memorized. The image is divided into 4 blocks to compress and encrypt. Then random pixel exchanging is introduced to scramble the compressed and encrypted blocks. Compared with the methods adopting the whole measurement matrix as key, the proposed algorithm shortens the key greatly, which is of importance for a practical encryption algorithm. By utilizing the circulate matrix to construct the measurement matrix, in CS and controlling the original row vector of circulate matrix with chaos system, the proposed algorithm is secure. By introducing the random pixel exchanging and binding the random matrices with the measurement matrices, the security is enhanced further. The simulation results show that this image compression encryption hybrid algorithm can provide good security and nice compression performance.

Vijayvargiya et al. [6] explain the main goal of image compression is to exemplify an image in the smallest number of bits without losing the major information content within an original image Compression methods are being speedily improved for compress large data files like images, there are several algorithms which perform this compression in various ways; some of these compression methods are designed for the specific type of images, thus they will not be perfect for other kinds of images, this study addresses about different image compression method. In this study they look over various kinds of current procedure of image compression such as Inter Pixel Redundancy where about in image adjacent pixels are not statistically separate, it is according to the connection between the neighboring pixels of an image, this kind of redundancy is known as Inter-pixel redundancy, this kind of redundancy may also referred to as spatial redundancy, this redundancy may be examine in many ways, one of which is through expecting a pixel value depend on the values of its adjacent pixels. In order to do so, the original 2-D array of pixels is generally mapped into a various shape.

The JPEG has been suggested as a standard compression scheme for continuous-tone motionless images. It utilizes a 64 $(8 \times 8)$ pixel-block discrete cosine transform $(\mathrm{DCT})$ for gath- ering the information into several transform coefficients, this block design takes advantage of the local spatial correlation property of images and also reduce the processing time. Yet, it is well known that this individual processing of each block will create visually disturbed blocking effects, especially when a high quantization parameter is used for high compression [7].

Otair and Shehadeh [4] proposed a novel lossy image compression technique called RIFD for compressing images. This scheme leans on increasing the redundancy and resemblance among the close pixels of images by rounding the pixels' intensities followed by the dividing process, which makes compression attainable, the main idea of the RIFD algorithm is based on two facts: 1) adjacent pixels are correlated or so identical; 2) the human sight can perceive a very limited number of intensities. So, if the intensity values of the adjacent pixels are rounding to the same value, then the redundancy will increase, and the updated intensity values will not be detectable by human sights. Raising the information redundancy supports the image to be more compressed. Therefore, finding a less correlated representation of the image is a significant thing. This technique can be implemented either individual or along with any lossless compression algorithm such as Huffman. The RIFD technique can be implemented via very simple steps as follow: 1) estimate image size; 2) rounding each pixel value to the nearest ten; 3) divide the rounded values by ten; and 4) apply Huffman Technique. This sequence aims to reduce the range of the intensities, as well as increasing intensities redundancy, which achieves better compression performance. A significant performance of RIFD technique remarked when it is followed by Huffman algorithm.

\section{OBJECTIVE AND EVAluation MEtrics}

Important image waste of information or property that may take place during the different image processing; Therefore, Image Quality Assessment (IQA) is deeply essential characteristic for evaluating image quality after been processed compared with the original image to ensure that any particular process is performing the required results, as in image compression it required to check the variation between the original and the processed Image [8]. IQA techniques could be: Objective or Subjective. Subjective evaluation is timeconsuming, costly and resource-intensive, this type gets along with human visual system (HVS) [9]. Objective image feature metrics can be grouped according to the availability of an original (distortion-free) image, in which the distorted image is to be compared. The current measures that will be used in this paper includes [10], [11]: Mean Square Error (MSE), Peak Signal to Noise Ratio (PSNR), Mean Absolute Error (MAE).

\section{A. Mean Square Error (MSE)}

The cumulative difference between the compressed image and original image:

$$
M S E=\frac{\sum_{m, n}\left[I_{1}(m, n)-I_{2}(m, n)\right]^{2}}{m \times n}
$$

Where, $\mathrm{M}, \mathrm{N}$ are pixel co-ordinate, I1: compressed image pixel, and I2: original image pixel. 


\section{B. Peak Signal to Noise Ratio (PSNR)}

The rate within largest possible power and effective distorted noise on image impersonation:

$$
P S N R=\frac{10 \times \log _{10}\left(\text { Intensity }_{(\max )}\right)^{2}}{M S E}
$$

For 8-bit pixel gray scale, Intensity $(\max )=255$,

$$
P S N R=\frac{10 \times \log _{10} 255^{2}}{M S E}
$$

\section{Mean Absolute Error (MAE)}

The metric represent the cumulative absolute value for the variance between the initial image and the refined one:

$$
M A E=\frac{\sum_{m, n}\left|I_{1}(m, n)-I_{2}(m, n)\right|}{m \times n}
$$

Where, $\mathrm{M}, \mathrm{N}$ are pixel co-ordinate, I1: compressed image pixel, and I2: original image pixel.

\section{Proposed Technique (Minimizing Decreasing TECHNIQUE (MDT))}

This paper proposed a new lossless pre-processing technique that enhances the lossy techniques by producing a minimized distortion rates in the compressed. For this purpose two existing lossy techniques were selected as follow: the classical JPEG compression technique and new novel lossy technique called RIFD. The core idea of the proposed technique (which is called MDT) depends on minimizing the pixels values by decreasing/subtracting the minimum values from each row, column, and $2 \times 2$ block, respectively. The values of resulted pixels (after the decreasing steps were implemented) will be minimized and then could be represented by the minimum numbers of bits. MDT reduces the distortion and could enhance the compression ratio at the same time. The following steps are the detailed steps of the proposed compression phase:

1) Find the minimum pixel value for each row in the image(Store them into a one-dimensional array called MinRowArray).

2) Subtract every minimum value in MinRowArray from each pixel of its corresponding row.

3) Find the minimum pixel value for each column in the image (Store them into a one-dimensional array called MinColArray).

4) Subtract every minimum value in MinColArray from each pixel of its corresponding column.

5) Find the minimum value of the $2 \times 2$ block in the image (Store them into a two-dimensional array called MinBlockArray).

6) Subtract the minimum value in MinBlockArray from each pixel of its corresponding block.

7) Implement the compression phase of a Lossy technique.

The proposed technique tries to keep every bit value and by implementing a lossy technique a distortion will be minimized and it could improve the compression ratio in most images. These pre-process compression steps use four arrays: one for the compressed image and the three additional arrays to preserve the minimum pixels values from rows, columns, and $2 \times 2$ blocks, respectively. Reversing the compression process in the correct sequence will be executed as follows:

1) Implement the decompression phase of the lossy technique.

2) Add the minimum value in MinBlockArray to each pixel of its corresponding block.

3) Add the minimum value in MinColArray to each pixel of its corresponding column.

4) Add the minimum value in MinRowArray to each pixel of its corresponding row.

\section{A. How the Proposed Techniques MDT Does Work?}

In order to explain how MDT does work, consider the following $10 \times 10$ block that taken from a well-known image 'Cameraman'(see Fig. 1).

\begin{tabular}{|c|c|c|c|c|c|c|c|c|c|}
154 & 195 & 44 & 11 & 10 & 148 & 229 & 188 & 134 & 152 \\
\hline 153 & 189 & 58 & 15 & 18 & 201 & 229 & 215 & 90 & 150 \\
\hline 37 & 46 & 29 & 22 & 27 & 91 & 68 & 63 & 42 & 121 \\
\hline 12 & 12 & 13 & 16 & 17 & 37 & 26 & 11 & 20 & 109 \\
\hline 13 & 12 & 11 & 13 & 15 & 25 & 25 & 11 & 13 & 74 \\
\hline 20 & 12 & 9 & 9 & 9 & 8 & 8 & 8 & 8 & 28 \\
\hline 17 & 11 & 10 & 9 & 8 & 8 & 9 & 8 & 9 & 12 \\
\hline 15 & 11 & 9 & 9 & 9 & 9 & 9 & 8 & 10 & 10 \\
\hline 15 & 12 & 10 & 14 & 39 & 32 & 18 & 9 & 9 & 10 \\
\hline 12 & 17 & 11 & 79 & 186 & 178 & 87 & 15 & 14 & 17 \\
\hline
\end{tabular}

Fig. 1. 10X10 block from Cameraman image.

Fig. 2 shows the result of the implementation the first four steps of MDT. The aim of these steps is to normalize pixels' values and increase the information redundancy which helps us to compress the image effectively (see Fig. 2).

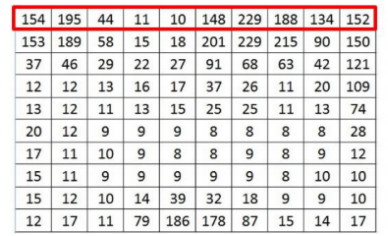

(a)

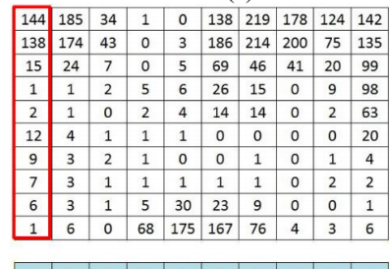

(c)

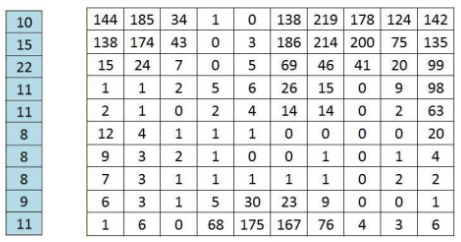

(b)

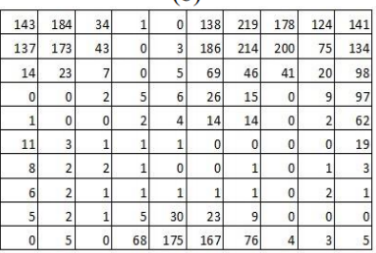

(d)
Fig. 2. (a) Find the minimum pixel value in every row, (b) Subtract the minimum row element from each pixel of its corresponding row, (c) Find the minimum pixel value in every column, (d) Subtract every minimum column element from each pixel of its corresponding column.

Fig. 3 shows the effect of implementing Steps 5 and 6 of MDT. The last two steps make the pixel values are ready to be compressed with any existing lossy compression technique 
and preserve the quality compressed images at the same time (see Fig. 3).

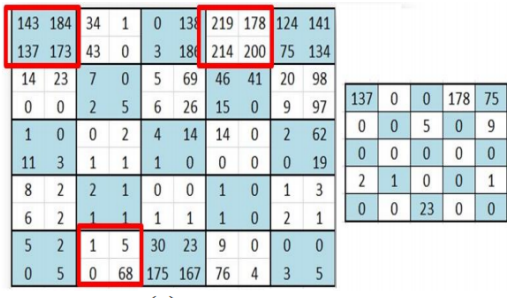

(a)

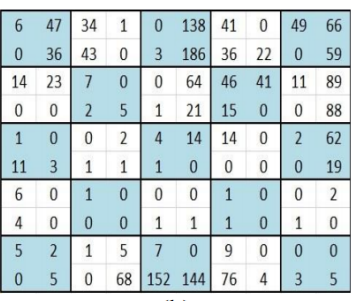

(b)
Fig. 3. (a) Find the minimum pixel value in every $2 \mathrm{X} 2$ block, (b) Subtract the minimum $2 \times 2$ block element from each pixel of its corresponding block.

Fig. 4 show the effect of implementing the RIFD steps on the last block from Fig. 3(b), which rounds every pixel to the nearest ten and then divide them by 10 . Finally, the range of the pixels' values fall between 0 and 19 (as in Fig. 4(b)). Thus, every pixel could be represented by 5 bits rather than 8 bits.

\begin{tabular}{|c|c|c|c|c|c|c|c|c|c|}
\hline 10 & 50 & 30 & 0 & 0 & 140 & 40 & 0 & 50 & 70 \\
\hline 0 & 40 & 40 & 0 & 0 & 190 & 40 & 20 & 0 & 60 \\
\hline 10 & 20 & 10 & 0 & 0 & 60 & 50 & 40 & 10 & 90 \\
\hline 0 & 0 & 0 & 0 & 0 & 20 & 10 & 0 & 0 & 90 \\
\hline 0 & 0 & 0 & 0 & 0 & 10 & 10 & 0 & 0 & 60 \\
\hline 10 & 0 & 0 & 0 & 0 & 0 & 0 & 0 & 0 & 20 \\
\hline 10 & 0 & 0 & 0 & 0 & 0 & 0 & 0 & 0 & 0 \\
\hline 0 & 0 & 0 & 0 & 0 & 0 & 0 & 0 & 0 & 0 \\
\hline 0 & 0 & 0 & 0 & 10 & 0 & 10 & 0 & 0 & 0 \\
\hline 0 & 0 & 0 & 70 & 150 & 140 & 80 & 0 & 0 & 0 \\
\hline
\end{tabular}

(a)

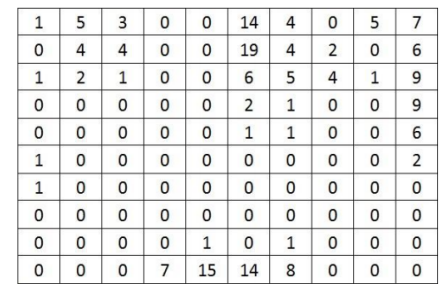

(b)
Fig. 4. (a) Rounding the Pixels' values to the nearest ten, (b) Dividing the Pixels' values by ten.

This scheme leans on increasing the redundancy and resemblance among the close pixels of images by rounding the pixels' intensities followed by the dividing process, which makes compression attainable.

\section{EXPERIMENTS}

In order to prove that MDT (the proposed pre-process compression steps) is suitable and efficient to be implemented before any lossy technique, two techniques were chosen as samples to achieve this purpose. The researchers of this paper chosen RIFD which is a simple and modern compression technique that depends on rounding and dividing steps (Hybrid: MDT-RIFD), and also the MDT was implemented with JPEG that performs complicated mathematical operations to compress the images (Hybrid: MDT-JPEG). All the techniques are written and implemented using Matlab2015a.

The researchers create an image set of 46 images: 30 gray-scale (14 images with 8-bit and 16 images with 16-bit depth) and 16 colored (RGB). These images are diverse in the types: GIF, TIF, PNG, and JPG. Moreover, they have different dimensions that varied from $120 \times 120$ to $1880 \times 1200$. In the image compression field the lossy compression techniques are evaluated using: Subjective and Objective measures. The subjective evaluation is achieved by human observer who sentences the quality of the decompressed images.

\section{A. Subjective Evaluation Experiments}

The purpose of this subsection is prove efficiency of the proposed two hybrid techniques MDT-RIFD and MDT-JPEG from the subjective evaluation perspective. Fig. 5 shows the decompression process on two images from the image set of gray-scale 8-bit images. It is noticeable that the quality of the decompressed images using the two proposed techniques are very high and acceptable. Fig. 6 shows the decompression process on other two colored images from the image set. Once again, it is noticeable that the quality of the decompressed colored images using the two proposed techniques are very high and acceptable.

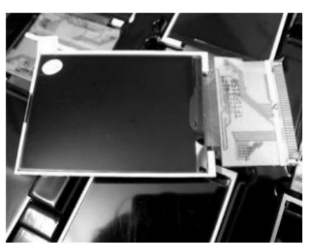

(a)

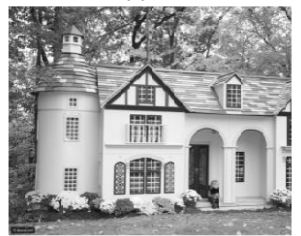

(c)

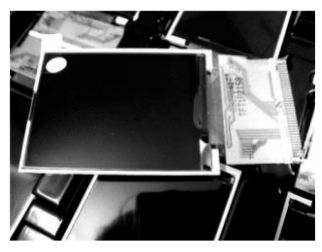

(b)

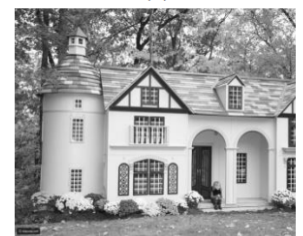

(d)
Fig. 5. Quality of Grayscale Image compression/Decompression using MDTRIFD and MDT-JPEG: (a) Original Image-1, (b) De-compressed Image-1 using MDT-RIFD, (c) Original Image-2, (d) De-compressed Image-2 using MDT-JPEG

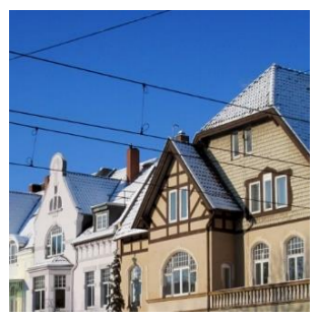

(a)

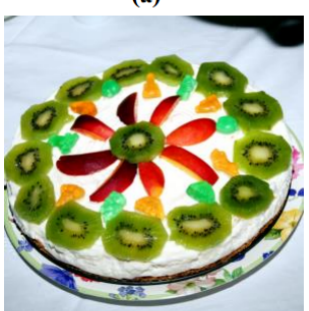

(c)

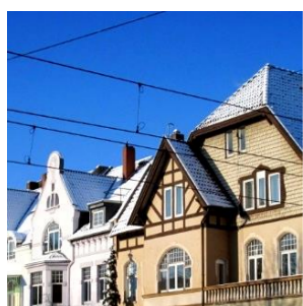

(b)

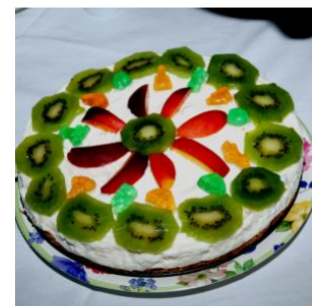

(d)
Fig. 6. Quality of Color(RGB) Image compression/Decompression using MDT-RIFD and MDT-JPEG: (a) Original Image-1, (b) De-compressed Image1 using MDT-RIFD, (c) Original Image-2, (d) De-compressed Image-2 using MDT-JPEG.

\section{B. Objective Evaluation Experiments}

The objective evaluation measures (MSE, MAE, and PSNR as discussed in Section 3) were used in the objective experiments. The hybrid proposed techniques MDT-RIFD and MDT- 
JPEG have been tested over all the image set, and then they were compared with the original RIFD and JPEG, respectively.

1) Experiments on 8-bits images: The purpose of the first part of the experiments is to test the hybrid proposed techniques MDT-RIFD and MDT-JPEG over the 8-bit images (labeled from g1 to g14). Table I compares RIFD and MDTRIFD in terms of: MSE, MAE, PSNR, and Compression Ratio (CR). The compression ratio is computed using the following formula:

$$
C r=\frac{\text { compressed image size }}{\text { original image size }}
$$

The results show the superiority of MDT-RIFD over the standard RIFD with all tested images based on the used measures and the compression ratio. The results of the used quality measures, MSE and MAE must be least as much as possible. At the other hand, PSNR is preferred to be larger. So, the hybrid MDT-RFID achieved these goals with all tested images. The improvement percentage for each quality metric (MSE, MAE and PSNR) for each image will be computed based on the following formula:

$$
\begin{gathered}
\text { Enhancement }= \\
\frac{(M S E, M A E \text { or } P S N R \text { of } R I F D)-(M S E, M A E \text { or } P S N R \text { of } M D T-R I F D)}{(M S E, M A E, \text { or PSNR of RIFD })}
\end{gathered}
$$

Note: this formula was used to compare the achieved enhancement (in all experiments) using the proposed MDT-RIFD and MDT-JPEG with the classical ones in terms of MSE, MAE, and PSNR. For example the achieved enhancement of resulted MSE for image g3.png is computed as follows:

$$
\begin{gathered}
\text { Enhancement }= \\
\frac{(M S E \text { using RIFD })-(M S E \text { using MDT }- \text { RIFD })}{(M S E \text { using RIFD })} \\
\text { Enhancementfor }(\text { g3.png })=\frac{(8.34)-(6.39)}{(8.34)}
\end{gathered}
$$

MSE Enhancement for g3.png $=0.23$, which is the least achieved enhancement with all tested 8-bit images. At the other hand, the best achieved enhancement of MSE was with image g5.gif which is equal to 0.70. The remaining enhancement results for MAE and PSNR were as follows:

- $\quad$ MAE: from $21 \%$ (g10.tif) to $63 \%$ (g5.png)

- PSNR: from 3\% (g3.png, g11.jpg, g14.jpg) to $14 \%$ (g5.png)

Fig. 7 is a graphical representation of Table I, which compares the results of RIFD and MDT-RIFD on 8-bit images. $\mathrm{X}$-coordinate represents the image number, and Y-coordinate are: (a) MSE, (b) MAE, (c) PSNR, and (d) Compression Ratio results, respectively ( $\mathrm{X}$ and $\mathrm{Y}$ coordinates in the rest figures are identical to Fig. 7). The experimental results show that MDTRIFD enhances the quality of all decompressed 8-bit images. Moreover, it remains or enhances the compression ratio for 12 of 14 (of the tested images), which means that the proposed technique enhances the quality and the efficiency at the same time.
TABLE I. COMPARATIVE BETWEEN RIFD AND MDT-RIFD ON 8-BIT IMAGES

\begin{tabular}{|c|c|c|c|c|c|c|c|c|}
\hline & \multicolumn{3}{|c|}{ MSE } & \multicolumn{2}{|c|}{ MAE } & \multicolumn{2}{|c|}{ PSNR } & \multicolumn{2}{|c|}{ CR } \\
\hline Image & RIFD & MDT-RIFD & RIFD & MDT-RIFD & RIFD & MDT-RIFD & RIFD & MDT-RIFD \\
\hline g1.gif & 9.93 & 4.35 & 3.1 & 1.33 & 38.19 & 41.78 & 0.48 & 0.46 \\
\hline g2.gif & 8.53 & 6.52 & 2.63 & 1.9 & 38.86 & 40.02 & 0.54 & 0.51 \\
\hline g33.png & 8.34 & 6.39 & 2.6 & 1.88 & 38.95 & 40.11 & 0.49 & 0.49 \\
\hline g4.gif & 8.53 & 5.36 & 2.62 & 1.66 & 38.86 & 40.88 & 0.48 & 0.47 \\
\hline g5.png & 11.87 & 3.61 & 3.21 & 1.18 & 37.42 & 42.59 & 0.49 & 0.41 \\
\hline g6.jpg & 8.77 & 5.71 & 3.08 & 1.74 & 38.74 & 40.6 & 0.44 & 0.45 \\
\hline g7.tif & 8.49 & 6.04 & 2.67 & 1.81 & 38.88 & 40.35 & 0.52 & 0.44 \\
\hline g8.png & 7.76 & 5.23 & 2.63 & 1.57 & 39.27 & 40.98 & 0.43 & 0.46 \\
\hline g9.png & 8.57 & 4.6 & 2.69 & 1.44 & 38.84 & 41.54 & 0.55 & 0.49 \\
\hline g10.tif & 8.71 & 5.96 & 2.28 & 1.79 & 38.76 & 40.41 & 0.51 & 0.44 \\
\hline g11.jpg & 8.45 & 6.3 & 3.24 & 1.86 & 38.89 & 40.17 & 0.55 & 0.53 \\
\hline g12.gif & 8.43 & 6.02 & 3.24 & 1.81 & 38.9 & 40.37 & 0.53 & 0.51 \\
\hline g13.png & 8.32 & 5.87 & 2.51 & 1.76 & 38.96 & 40.48 & 0.55 & 0.54 \\
\hline g14.jpg & 8.41 & 6.5 & 2.53 & 1.9 & 38.92 & 40.04 & 0.58 & 0.53 \\
\hline
\end{tabular}

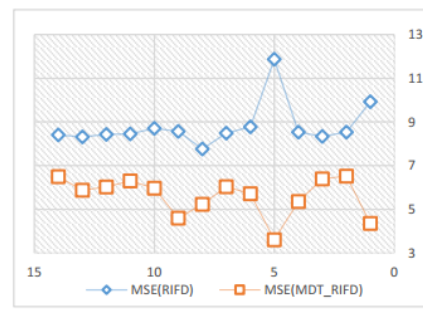

(a)

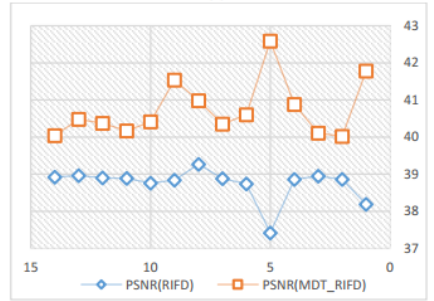

(c)

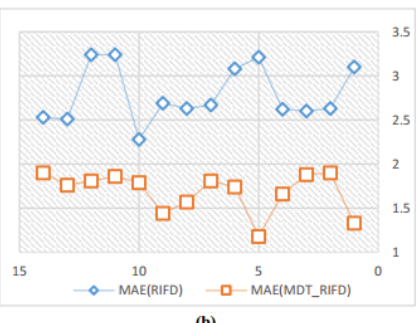

(b)

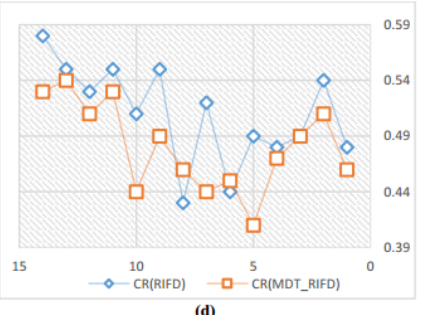

Fig. 7. Comparative between RIFD and MDT-RIFD on 8-bit Images:(a) MSE results, (b) MAE results, (c) PSNR results, (d) Compression Ratio Results.

Table II compares between JPEG and MDT-JPEG on the 8-bit image set. MDT-JPEG produced better results over the standard JPEG with all images according to the used measures and the compression ratio. The achieved enhancements were as follows:

- $\quad$ MSE: from $11 \%$ (g13.png) to 84\% (g5.png)

- MAE: from 24\% (g1.gif) to $97 \%$ (g10.tif)

- PSNR: from 1\% (g11.jpg) to $24 \%$ (g5.png)

Fig. 8 is a graphical representation of Table II (it compares the results between JPEG and MDT-JPEG on 8-bit images). The curves show that MDT-JPEG enhances the quality of all decompressed 8-bit images. Moreover, it enhances the compression ratio with all images except a single image.

2) Experiments on 16-bits images: The purpose of the second part of the experiments is to test the hybrid proposed techniques MDT-RIFD and MDT-JPEG over the 16-bit images (numbered from 1 to 16). Table III compares RIFD and MDTRIFD in terms of: MSE, MAE, PSNR, and Compression Ratio. MDT-RIFD gives better results in compare with the standard RIFD along all tested 16-bit images based on the used measures and the compression ratio. The achieved enhancements were as follows: 
TABLE II. COMPARATIVE BETWEEN JPEG AND MDT-JPEG ON 8-BIT IMAGES

\begin{tabular}{|c|c|c|c|c|c|c|c|c|}
\hline & \multicolumn{2}{|c|}{ MSE } & \multicolumn{2}{|c|}{ MAE } & \multicolumn{2}{|c|}{ PSNR } & \multicolumn{2}{c|}{ CR } \\
\hline Image & JPEG & MDT-JPEG & JPEG & MDT-JPEG & JPEG & MDT-JPEG & JPEG & MDT-JPEG \\
\hline g1.gif & 82.24 & 60.48 & 12.12 & 9.21 & 29.01 & 30.35 & 0.33 & 0.38 \\
\hline g2.gif & 255 & 86.06 & 143.73 & 6.04 & 24.1 & 28.82 & 0.36 & 0.42 \\
\hline g3.png & 68.87 & 51.32 & 6.98 & 5.19 & 29.78 & 31.06 & 0.31 & 0.43 \\
\hline g4.gif & 150.13 & 60.96 & 64.75 & 4.44 & 26.4 & 30.31 & 0.33 & 0.40 \\
\hline g5.png & 186.57 & 30.76 & 33.11 & 2.89 & 25.46 & 33.28 & 0.25 & 0.29 \\
\hline g6.jpg & 119.79 & 112.04 & 33.55 & 6.45 & 27.38 & 27.67 & 0.3 & 0.37 \\
\hline g7.tif & 254.98 & 81.22 & 136 & 5.45 & 24.1 & 29.07 & 0.31 & 0.33 \\
\hline g8.png & 254.43 & 114.38 & 164.69 & 6.92 & 24.11 & 27.58 & 0.37 & 0.39 \\
\hline g9.png & 147.75 & 95.37 & 58.97 & 5.54 & 26.47 & 28.37 & 0.33 & 0.45 \\
\hline g10.tif & 145.99 & 58.77 & 181.47 & 4.54 & 26.52 & 30.47 & 0.39 & 0.37 \\
\hline g11.jpg & 103.53 & 97.19 & 29.24 & 6.68 & 28.01 & 28.29 & 0.39 & 0.43 \\
\hline g12.gif & 117.24 & 93.46 & 13.14 & 6.32 & 27.47 & 28.46 & 0.40 & 0.48 \\
\hline g13.png & 144.33 & 129.08 & 59.09 & 7.62 & 26.57 & 27.06 & 0.38 & 0.54 \\
\hline g14.jpg & 116.31 & 101.33 & 37.28 & 8.88 & 27.51 & 28.11 & 0.34 & 0.51 \\
\hline
\end{tabular}

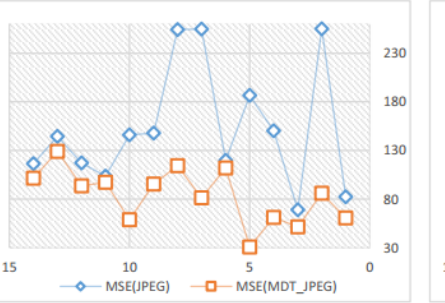

(a)

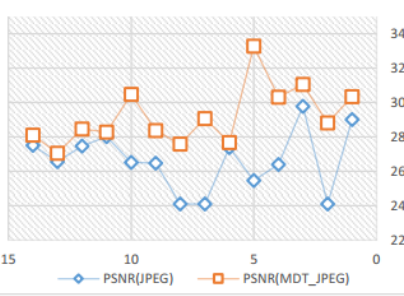

(c)

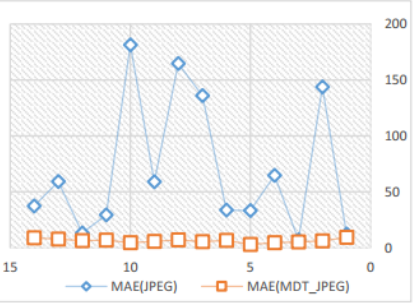

(b)

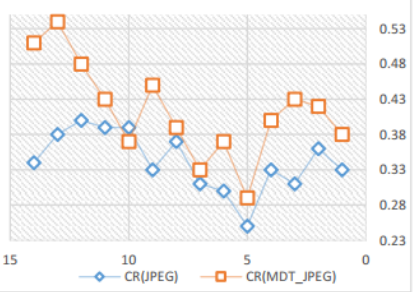

(d)
Fig. 8. Comparative between JPEG and MDT-JPEG on 8 bit Images: (a) MSE results, (b) MAE results, (c) PSNR results, (d) Compression Ratio Result.

- MSE: from $10 \%$ (15.png) to $43 \%$ (10.gif, 11.png, 13.tif, 14.tif)

- MAE: from $11 \%$ (15.png) to $69 \%$ (14.tif)

- PSNR: from 10\% (15.png) to 43\% (10.gif, 11.png, 13.tif, 14.tif)

Fig. 9 is a graphical representation of Table III (it compares the results of RIFD and MDT-RIFD on 16-bit images). The curves show that MDT-RIFD enhances the quality of all decompressed 16-bit images. Moreover, it enhances the compression ratio with all tested images.

TABLE III. COMPARATIVE BETWEEN RIFD AND MDT-RIFD ON 16-BIT IMAGES

\begin{tabular}{|c|c|c|c|c|c|c|c|c|}
\hline & \multicolumn{2}{|c|}{ MSE } & \multicolumn{2}{c|}{ MAE } & \multicolumn{2}{|c|}{ PSNR } & \multicolumn{2}{|c|}{ CR } \\
\hline Image & RIFD & MDT-RIFD & RIFD & MDT-RIFD & RIFD & MDT-RIFD & RIFD & MDT-RIFD \\
\hline 1.gif & 21,869 & 18,065 & 125.96 & 103.17 & 47.64 & 53.76 & 0.35 & 0.41 \\
\hline 2.gif & 21,671 & 17,234 & 125.8 & 98.84 & 47.67 & 53.97 & 0.37 & 0.45 \\
\hline 3.png & 21,603 & 18,154 & 125.18 & 104.81 & 45.04 & 53.74 & 0.35 & 0.41 \\
\hline 4.png & 21,487 & 18,669 & 124.53 & 107.55 & 47.71 & 53.62 & 0.24 & 0.40 \\
\hline 5.png & 21,470 & 17,192 & 124.44 & 99.37 & 46.65 & 53.98 & 0.33 & 0.46 \\
\hline 6.png & 21,277 & 16,996 & 123.26 & 98.4 & 48.81 & 54.03 & 0.35 & 0.44 \\
\hline 7.gif & 21,053 & 16,457 & 122.15 & 95.29 & 45.13 & 54.17 & 0.35 & 0.42 \\
\hline 8.png & 21,859 & 17,876 & 126.43 & 103.9 & 37.05 & 53.81 & 0.31 & 0.39 \\
\hline 9.png & 21,490 & 18,997 & 124.85 & 109.35 & 45.06 & 53.54 & 0.31 & 0.38 \\
\hline 10.gif & 15,510 & 10,840 & 90.12 & 65.04 & 46.26 & 55.98 & 0.31 & 0.40 \\
\hline 11.png & 21,536 & 15,109 & 124.81 & 80.41 & 45.05 & 54.54 & 0.35 & 0.47 \\
\hline 12.png & 18,150 & 15,089 & 110.19 & 74.22 & 46.75 & 54.54 & 0.17 & 0.43 \\
\hline 13.tif & 20,678 & 14,447 & 117.69 & 72.78 & 45.2 & 54.73 & 0.35 & 0.44 \\
\hline 14.tif & 21,042 & 14,732 & 119.22 & 70.62 & 48.32 & 54.65 & 0.31 & 0.41 \\
\hline 15.png & 21,388 & 19,372 & 123.9 & 11.45 & 45.07 & 53.46 & 0.36 & 0.37 \\
\hline 16.png & 21,617 & 17,613 & 125.27 & 101.9 & 47.15 & 53.87 & 0.28 & 0.40 \\
\hline
\end{tabular}

TABLE IV COMPARATIVE BETWEEN JPEG AND MDT-JPEG ON 16-BIT IMAGES

\begin{tabular}{|c|c|c|c|c|c|c|c|c|}
\hline & \multicolumn{2}{|c|}{ MSE } & \multicolumn{2}{|c|}{ MAE } & \multicolumn{2}{|c|}{ PSNR } & \multicolumn{2}{c|}{ CR } \\
\hline Image & JPEG & MDT-JPEG & JPEG & MDT-JPEG & JPEG & MDT-JPEG & JPEG & MDT-JPEG \\
\hline 1.gif & 32,193 & 22,404 & 18,819 & 680 & 51.25 & 52.83 & 0.25 & 0.40 \\
\hline 2.gif & 39,249 & 22,804 & 23,832 & 912 & 50.39 & 52.75 & 0.32 & 0.43 \\
\hline 3.png & 24,193 & 21,506 & 11,572 & 624 & 52.49 & 53 & 0.25 & 0.40 \\
\hline 4.png & 23,637 & 20,637 & 1261 & 592 & 52.59 & 53.18 & 0.29 & 0.43 \\
\hline 5.png & 32,696 & 26,696 & 3313 & 1391 & 51.18 & 52.07 & 0.35 & 0.46 \\
\hline 6.png & 28,447 & 23,447 & 6050 & 934 & 51.79 & 52.63 & 0.32 & 0.44 \\
\hline 7.gif & 39,181 & 32,179 & 7059 & 2306 & 50.4 & 51.25 & 0.41 & 0.34 \\
\hline 8.png & 21,480 & 19,480 & 3070 & 448 & 53.01 & 53.43 & 0.27 & 0.40 \\
\hline 9.png & 19,476 & 18,476 & 2875 & 364 & 53.43 & 53.66 & 0.27 & 0.40 \\
\hline 10.gif & 19,177 & 15,177 & 5577 & 716 & 53.5 & 54.52 & 0.28 & 0.40 \\
\hline 11.png & 27,717 & 23,717 & 11,604 & 1052 & 51.9 & 52.58 & 0.34 & 0.45 \\
\hline 12.png & 32,814 & 23,513 & 22,802 & 860 & 51.17 & 52.62 & 0.24 & 0.42 \\
\hline 13.tif & 30,882 & 23,882 & 6030 & 943 & 51.43 & 52.55 & 0.32 & 0.44 \\
\hline 14.tif & 25,024 & 21,024 & 3150 & 545 & 52.35 & 53.1 & 0.30 & 0.41 \\
\hline 15.png & 31,659 & 19,271 & 5610 & 379 & 51.32 & 53.48 & 0.26 & 0.40 \\
\hline 16.png & 27,698 & 21,698 & 1680 & 583 & 51.91 & 52.97 & 0.29 & 0.42 \\
\hline
\end{tabular}

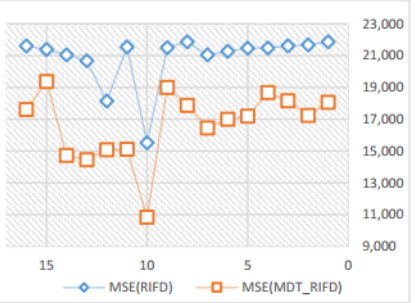

(a)

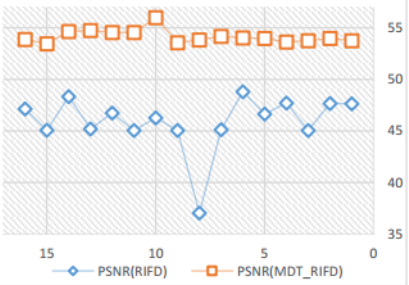

(c)
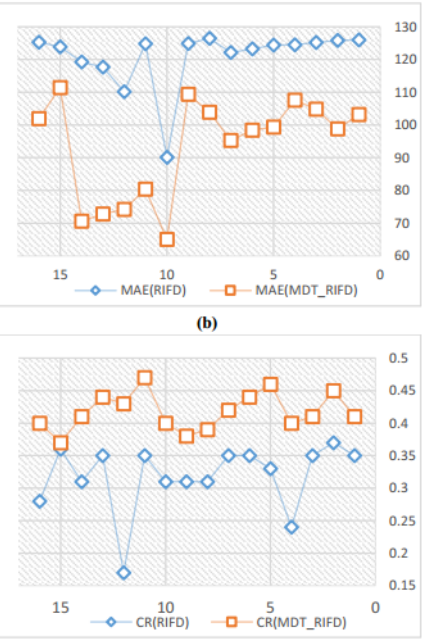

(d)
Fig. 9. Comparative between RIFD and MDT-RIFD on 16-bit Images: (a) MSE results, (b) MAE results, (c) PSNR results, (d) Compression Ratio Results.

Table IV compares JPEG and MDT-JPEG on the 16-bit image set in terms of: MSE, MAE, PSNR, and Compression Ratio. The results show the superiority of MDT-JPEG over the standard RIFD with all tested images based on the used measures and the compression ratio (except only one image). The achieved enhancements were as follows:

- MSE: from $11 \%$ (3.png) to $42 \%$ (2.gif)

- MAE: from 53\% (4.png) to 96\% (12.png)

- PSNR: from 1\% (3.png, 4.png, 8.png, 11.png, 14.tif) to $5 \%$ (2.gif)

Fig. 10 is a graphical representation of Table IV, which compares the results of JPEG and MDT- JPEG on 16-bit images. The curves show that MDT- JPEG enhances the quality of all decompressed 16-bit images. Moreover, it enhances the compression ratio with all tested images except a single image.

3) Experiments on colored images: The purpose of the second part of the experiments is to test the hybrid proposed techniques MDT-RIFD and MDT-JPEG over the colored 


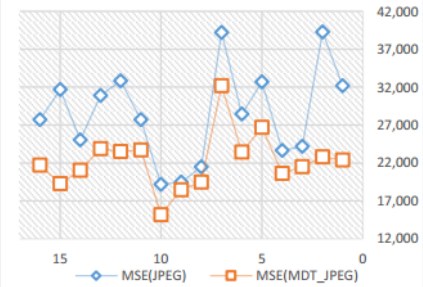

(a)

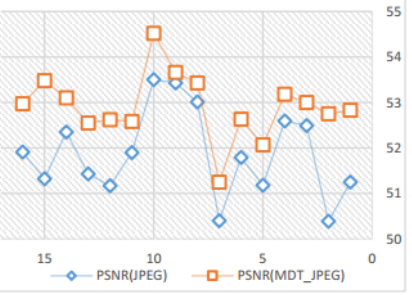

(c)
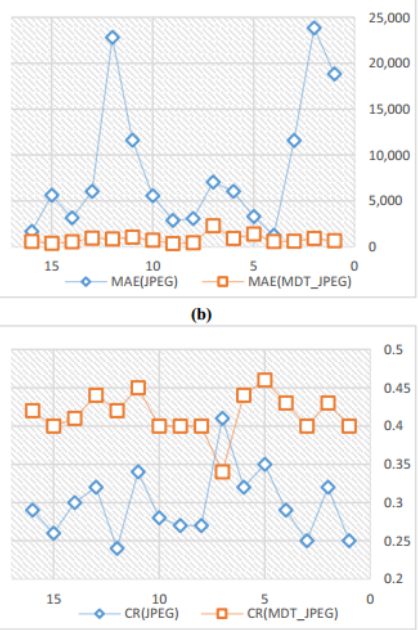

(d)
Fig. 10. Comparative between JPEG and MDT-JPEG on 16-bit Images: (a) MSE results, (b) MAE results, (c) PSNR results, (d) Compression Ratio Results.

TABLE V. COMPARATIVE BETWEEN RIFD AND MDT-RIFD ON COLOR (RGB) IMAGES

\begin{tabular}{|c|c|c|c|c|c|c|c|c|}
\hline & \multicolumn{2}{|c|}{ MSE } & \multicolumn{2}{c|}{ MAE } & \multicolumn{2}{|c|}{ PSNR } & \multicolumn{2}{|c|}{ CR } \\
\hline Image & RIFD & MDT-RIFD & RIFD & MDT-RIFD & RIFD & MDT-RIFD & RIFD & MDT-RIFD \\
\hline c1.jpg & 8.54 & 6.31 & 2.5 & 1.86 & 38.9 & 40.16 & 0.56 & 0.35 \\
\hline c2.png & 8.95 & 3.95 & 2.62 & 1.33 & 38.7 & 42.2 & 0.45 & 0.26 \\
\hline c3.png & 8.65 & 5.92 & 2.51 & 1.78 & 38.8 & 40.44 & 0.55 & 0.36 \\
\hline c4.jpg & 8.32 & 2.79 & 2.46 & 1.1 & 39 & 43.7 & 0.43 & 0.23 \\
\hline c5.png & 8.4 & 5.3 & 2.48 & 1.64 & 38.9 & 40.93 & 0.53 & 0.34 \\
\hline c6.jpg & 8.56 & 5.51 & 2.51 & 1.68 & 38.8 & 40.75 & 0.53 & 0.33 \\
\hline c7.png & 8.55 & 6.21 & 2.5 & 1.84 & 38.9 & 40.24 & 0.56 & 0.33 \\
\hline c8.jpg & 8.39 & 5.65 & 2.48 & 1.73 & 39.9 & 40.65 & 0.52 & 0.29 \\
\hline c9.jpg & 8.48 & 4.42 & 2.5 & 1.36 & 38.9 & 41.71 & 0.44 & 0.27 \\
\hline c10.tif & 8.81 & 4.92 & 2.47 & 1.52 & 38.7 & 41.25 & 0.52 & 0.28 \\
\hline c11.png & 8.46 & 6.34 & 2.48 & 1.86 & 38.9 & 40.15 & 0.58 & 0.44 \\
\hline c12.jpg & 8.59 & 5.96 & 2.52 & 1.79 & 38.8 & 40.41 & 0.54 & 0.37 \\
\hline c13.tif & 8.41 & 5.96 & 2.47 & 1.79 & 38.9 & 40.41 & 0.55 & 0.33 \\
\hline c14.png & 8.4 & 6.22 & 2.45 & 1.85 & 38.9 & 40.23 & 0.38 & 0.25 \\
\hline c15.tif & 9.14 & 3.73 & 2.59 & 1.31 & 38.6 & 42.45 & 0.48 & 0.24 \\
\hline c16.jpg & 8.71 & 6.42 & 2.50 & 1.89 & 38.8 & 40.09 & 0.55 & 0.28 \\
\hline
\end{tabular}

(RGB) images (labeled from c1 to c16). Table V compares RIFD and MDT-RIFD in terms of: MSE, MAE, PSNR, and Compression Ratio. The results show the superiority of MDTRIFD over the standard RIFD with all tested colored (RGB) images based on the used measures and the compression ratio. The achieved enhancements were as follows:

- MSE: from 25\% (c11.png) to 66\% (c4.jpg)

- MAE: from 25\% (c11.png) to 55\% (c4.jpg)

- $\quad$ PSNR: from 3\% (c1.jpg, c11.png, c14.png, c16.jpg) to $12 \%$ (c4.jpg)

Fig. 11 is a graphical representation of Table V, which compares the results of RIFD and MDT-RIFD on colored (RGB) images. The curves show significant quality enhancement of all decompressed colored (RGB) images using MDTRIFD. Moreover, it enhances the compression ratio for all tested images. Table VI compares JPEG and MDT-JPEG on the colored (RGB) image set in terms of: MSE, MAE, PSNR, and Compression Ratio. The results show the superiority of MDT-JPEG over the standard JPEG with all tested images based on the used measures. The achieved enhancements were as follows:
TABLE VI. COMPARATIVE BETWEEN JPEG AND MDT-JPEG ON COLOR (RGB) IMAGES

\begin{tabular}{|c|c|c|c|c|c|c|c|c|}
\hline & \multicolumn{2}{|c|}{ MSE } & \multicolumn{2}{c|}{ MAE } & \multicolumn{2}{c|}{ PSNR } & \multicolumn{2}{c|}{ CR } \\
\hline Image & JPEG & MDT-JPEG & JPEG & MDT-JPEG & JPEG & MDT-JPEG & JPEG & MDT-JPEG \\
\hline c1.jpg & 119.53 & 9.66 & 8.64 & 1.26 & 27.39 & 38.32 & 0.3 & 0.26 \\
\hline c2.png & 185.84 & 5.71 & 14.12 & 0.99 & 25.47 & 40.6 & 0.32 & 0.54 \\
\hline c3.png & 120.98 & 9.98 & 7.21 & 1.21 & 27.34 & 38.17 & 0.22 & 0.14 \\
\hline c4.jpg & 98.96 & 1.96 & 8.61 & 0.61 & 28.21 & 45.24 & 0.14 & 0.46 \\
\hline c5.png & 172.56 & 9.56 & 14.98 & 1.22 & 25.8 & 38.36 & 0.49 & 0.46 \\
\hline c6.jpg & 221.79 & 11.32 & 11.17 & 1.48 & 24.71 & 37.63 & 0.46 & 0.54 \\
\hline c7.png & 144.24 & 14.24 & 12.46 & 1.81 & 26.57 & 36.63 & 0.57 & 0.33 \\
\hline c8.jpg & 160.2 & 6.36 & 13.73 & 0.95 & 26.12 & 40.13 & 0.37 & 0.28 \\
\hline c9.jpg & 290.66 & 4.25 & 15.41 & 0.75 & 23.53 & 41.88 & 0.27 & 0.35 \\
\hline c10.tif & 91.88 & 8.88 & 7.29 & 1.29 & 28.53 & 36.68 & 0.38 & 0.84 \\
\hline c11.png & 120.14 & 26.25 & 11.39 & 2.69 & 27.37 & 33.97 & 0.24 & 0.43 \\
\hline c12.jpg & 120.5 & 10.12 & 8 & 1.42 & 27.35 & 38.11 & 0.44 & 0.49 \\
\hline c13.tif & 95.38 & 9.55 & 8.99 & 1.21 & 28.37 & 38.36 & 0.55 & 0.15 \\
\hline c14.png & 173.28 & 4.66 & 18.23 & 1.04 & 25.78 & 41.48 & 0.18 & 0.18 \\
\hline c15.tif & 94.56 & 1.71 & 8.48 & 0.48 & 28.41 & 45.84 & 0.36 & 0.31 \\
\hline c16.jpg & 274.04 & 8.85 & 13.55 & 1.34 & 23.79 & 38.7 & 0.39 & 0.31 \\
\hline
\end{tabular}

- MSE: from 78\% (c11.png) to 99\% (c9.jpg)

- MAE: from $76 \%$ (c11.png) to $95 \%$ (c9.jpg)

- $\quad$ PSNR: from $24 \%$ (c11.png) to $78 \%$ (c9.jpg)

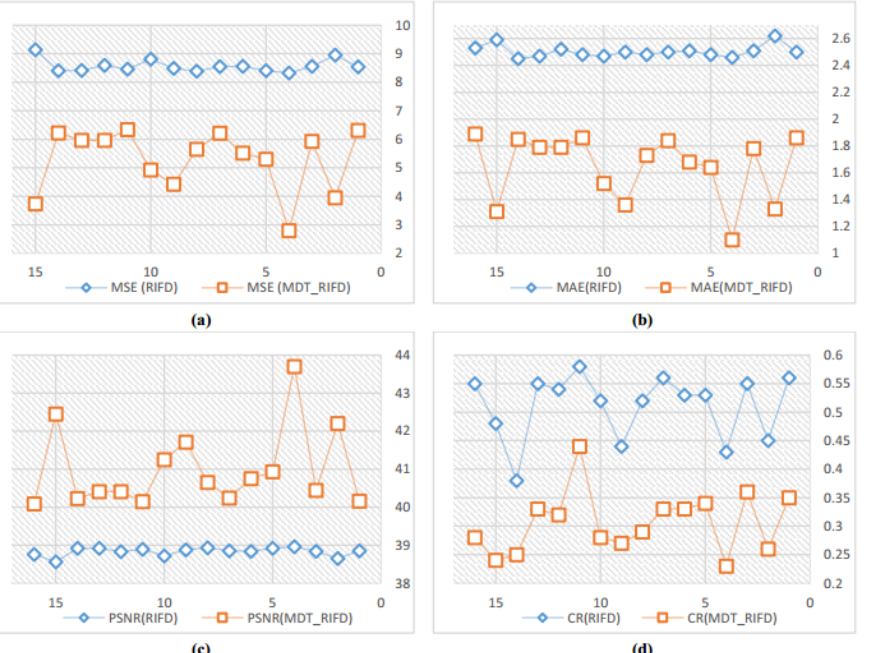

(d)

Fig. 11. Comparative between RIFD and MDT-RIFD on color(RGB) Images: (a) MSE results, (b) MAE results, (c) PSNR results, (d) Compression Ratio Results.

Fig. 12 is a graphical representation of Table VI, which compares the results of JPEG and MDT- JPEG on colored (RGB) images. The curves show that MDT-JPEG enhances the quality of all decompressed colored (RGB) images with significant percentage enhancement. However, it only enhances the compression ratio for $50 \%$ of the tested images.

\section{CONCLUSION}

This paper presented lossless pre-processing steps that could be generalized to be implemented before any lossy technique and was performed on various images that varied in types, dimension, and bit-depth. Two distinct lossy techniques were chosen RIFD and JPEG to be implemented with the proposed technique. These lossy techniques are vary from each other since RIFD is a novel/new compression technique that uses simple steps of rounding and dividing the pixels while JPEG is a well-known and widely used as a lossy 


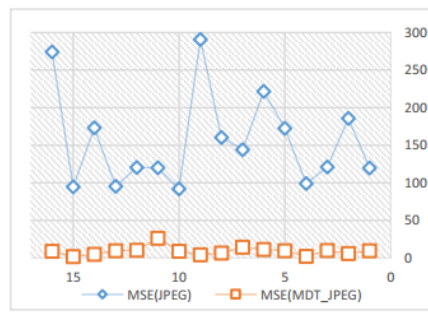

(a)

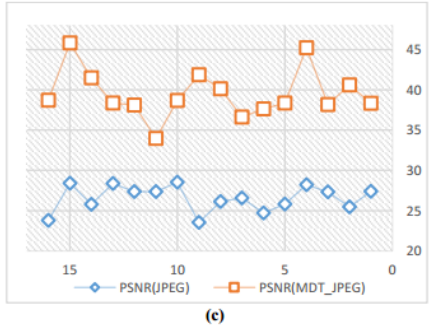

(c)

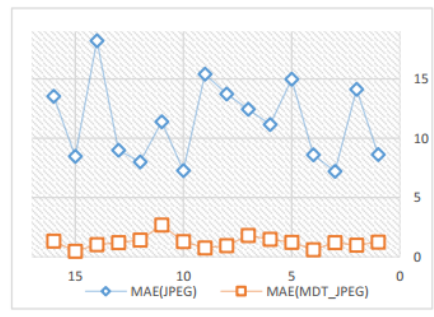

(b)

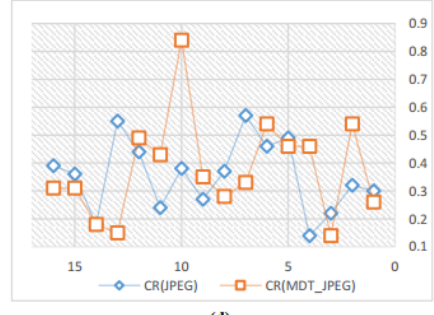

(d)
Fig. 12. Comparative between JPEG and MDT-JPEG on color (RGB) Images: (a) MSE results, (b) MAE results, (c) PSNR results, (d)Compression Ratio Results.

compression method which uses a complicated linear equations with discrete cosine transformation. The purpose of using these lossy techniques is to prove that the proposed technique can be applied along the most of the available and future lossy techniques. The results showed the superiority of MDT-RIFD and MDT-JPEG (over the standard RIFD and JPEG, respectively) in terms of the objective quality evaluation metrics such as: MSE, MAE, and PSNR. In the other hand, the hybrid techniques enhance the compression ratio with the most images. The achieved enhancements were varied from one image to another regarding the image characteristics, and the used lossy technique.

\section{REFERENCES}

[1] V. Hlavac, "Fundamentals of image processing," Optical and Digital Image Processing: Fundamentals and Applications, pp. 71-96, 2011.

[2] I. T. Young, J. J. Gerbrands, and L. J. Van Vliet, Fundamentals of image processing. Delft University of Technology Delft, 1998.

[3] M. M. H. Chowdhury and A. Khatun, "Image compression using discrete wavelet transform," IJCSI International Journal of Computer Science Issues, vol. 9, no. 4, pp. 327-330, 2012.

[4] M. Otair and F. Shehadeh, "Research article lossy image compression by rounding the intensity followed by dividing (rifd)," 2016.

[5] F. A. Jassim and H. E. Qassim, "Five modulus method for image compression," arXiv preprint arXiv:1211.4591, 2012.

[6] G. Vijayvargiya, S. Silakari, and R. Pandey, "A survey: various techniques of image compression," arXiv preprint arXiv:1311.6877, 2013.

[7] N. Zhou, A. Zhang, F. Zheng, and L. Gong, "Novel image compressionencryption hybrid algorithm based on key-controlled measurement matrix in compressive sensing," Optics \& Laser Technology, vol. 62, pp. 152-160, 2014.

[8] F. Huang, J. Huang, and Y.-Q. Shi, "New channel selection rule for jpeg steganography," IEEE Transactions on Information Forensics and Security, vol. 7, no. 4, pp. 1181-1191, 2012.

[9] T. Samajdar and M. I. Quraishi, "Analysis and evaluation of image quality metrics," in Information Systems Design and Intelligent Applications. Springer, 2015, pp. 369-378.

[10] M. Pedersen and J. Y. Hardeberg, "Full-reference image quality metrics: Classification and evaluation," Foundations and Trends $₫$ in Computer Graphics and Vision, vol. 7, no. 1, pp. 1-80, 2012.

[11] P. Kaushik and Y. Sharma, "Comparison of different image enhancement techniques based upon psnr \& mse," International Journal of Applied Engineering Research, vol. 7, no. 11, pp. 2010-2014, 2012. 